\title{
Força muscular em idosos II - Efeito de um programa comple- mentar de treino na força muscular de idosos de ambos os sexos
}

\author{
J. Carvalho \\ J. Oliveira \\ J. Magalhães \\ A. Ascensão \\ J. Mota \\ J.M.C. Soares
}

https://doi.org/10.5628/rpcd.04.01.58

\section{RESUMO}

O principal objectivo deste trabalho foi determinar o efeito de um programa combinado de actividade física na força máxima isocinética em idosos, homens e mulheres. Dezanove idosos, 12 mulheres (idade $=69.6 \pm 2.9$ anos; peso $=66.7 \pm 7.9 \mathrm{~kg}$; altura $=1.55 \pm 0.1 \mathrm{~m}$ ) e 7 homens (idade $=68.3 \pm 5.2$ anos; peso $=69.1 \pm 12.9 \mathrm{~kg}$; altura $=1.65 \pm 0.2 \mathrm{~m}$ ) participaram durante 6 meses num programa combinado de actividade física consistindo em 4 sessões semanais ( 2 x ginástica de manutenção e $2 \mathrm{x}$ treino de força). A força máxima isocinética dos extensores e flexores do joelho foi avaliada em todos os sujeitos através de um dinamómetro isocinético (Biodex System 2, USA) em duas velocidades distintas $60^{\circ} / \mathrm{seg}$. (1.05 rad.sec. $\left.{ }^{-1}\right)$ e $180^{\circ}$ /seg. (3.14 rad.sec ${ }^{-1}$ ) antes e depois do programa de treino. Os resultados deste estudo mostram que 6 meses de treino de força combinado com ginástica de manutenção é exequível e está associado a um aumento significativo da força muscular em idosos, particularmente no membro não-dominante. Para além disso, os resultados mostram que a variável sexo não influencia as adaptações induzidas pelo treino na força muscular. Apesar dos homens serem, em termos absolutos, mais fortes do que as mulheres, não foram observadas diferenças entre ambos os sexos na resposta ao treino. Assim, este estudo sugere que o treino progressivo de força com intensidade moderada a elevada pode ser efectuado com elevada tolerância por idosos saudáveis, desempenhando um papel importante enquanto estratégia para a manutenção e/ou aumento da sua força independentemente do sexo.

Palavras-chave: envelhecimento, força muscular, isocinético.

\author{
Faculdade de Ciências de Desporto e de Educação Física \\ Universidade do Porto, Portugal
}

\author{
ABSTRACT \\ Aging and muscle strength II - Effects of a combined physical \\ activity program in muscular strength in elderly.
}

The aim of the present study was to evaluate the effect of a combination of specific strength training plus a generalised physical activity program on knee extensor and flexor isokinetic peak torque of elderly adults according to gender. Nineteen elderly subjects, 12 women (age: years; weight: $66.7 \pm 7.9 \mathrm{~kg}$; height: $1.55 \pm 0.1 \mathrm{~m}$ ) and 7 men (age: $68.3 \pm 5.2$ years; weight: $69.1 \pm 12.9 \mathrm{~kg}$; height: $1.65 \pm 0.2 \mathrm{~m}$ ), participated in a 6-month program, consisting of 4 sessions per week (2 for generalized physical activity, 2 for strength training). All the subjects were tested for maximal strength of quadriceps and hamstrings on an isokinetic dynamometer (Biodex System 2, USA) at 60\% $/ \mathrm{sec}$. (1.05 rad.sec. ${ }^{-1}$ ) and $180^{\circ} / \mathrm{sec}\left(3.14 \mathrm{rad} . \mathrm{sec}^{-1}\right)$ before and after the training period. The results of this study showed that, a 6-month moderate-to-high-intensity strength training program combined with generalized physical activity is feasible and is associated with significant increasing in strength in older adults, namely on the non-dominant limb. Moreover, the results showed that gender does not influence the training induced adaptations in strength. In spite of men being stronger than women in absolute strength, there is no apparent difference between men and women in their responses to training. It seems reasonable that older adults, not only well tolerate moderate-to-high intensities of strength training, but also, this kind of physical activity programs can, independently of the gender, counteract the age-related decline in muscle strength.

Key Words: aging, muscle strength, isokinetic. 


\section{INTRODUÇÃO}

A redução da massa e da força muscular decorrentes do envelhecimento são dos aspectos mais frequentemente referidos na literatura $(1,14,17,22,24)$.

Esta fraqueza e a atrofia musculares, particularmente dos membros inferiores, tem sido associada ao maior risco de quedas (25), à diminuição da densidade mineral óssea (DMO) e à maior probabilidade de fracturas $(4,6)$, bem como, a outras alterações fisiológicas adversas, tais como, intolerância à glicose (28) e alterações no metabolismo energético e na capacidade aeróbia $(12,13)$.

Os músculos extensores e flexores do joelho desempenham um papel determinante na estabilidade corporal e na locomoção. O seu uso na marcha lenta e rápida (21), na subida de degraus (27) e no movimento de levantar da cadeira (29) tem sido bem documentado em estudos cinemáticos, de electromiografia e de análise cinética.

Numerosos trabalhos têm demonstrado que estímulos adequados de treino em idosos, independentemente do sexo, retardam a diminuição da força e da massa muscular normalmente associada ao envelhecimento $(15,19,20,22,30,36)$. Assim, programas com intensidade suficiente para aumentar a força e o equilíbrio devem ser implementados como forma de prevenção de quedas e lesões. Deste modo, porque em oposição a alguns autores $(25,32)$, não foram encontradas alterações significativas nos níveis de força dos idosos após implementação de um programa de actividade física generalizado (cf. estudo I), e considerando, tal como Fiatarone e Evans (11), a força muscular, particularmente a dos membros inferiores, como um factor limitador importante para a manutenção de um estilo de vida autónomo, o objectivo principal deste trabalho foi o de avaliar o efeito de um programa combinado de actividade física (i.e., "Ginástica de Manutenção + Musculação") sobre a força muscular dos membros inferiores do idoso em função da variável sexo.

\section{MATERIAL E MÉTODOS}

\section{Amostra}

A amostra inicial foi constituída por 34 idosos sedentários voluntários, com idades compreendidas entre os 65 e os 81 anos.
Todavia, 8 destes sujeitos foram excluídos das avaliações pelo facto de terem abandonado as sessões de treino específico de força ("Musculação"), mantendo-se apenas nas sessões de "Ginástica de Manutenção", 3 foram retirados, dada a não-presença a mais de $20 \%$ do total das sessões de actividade física e, por fim, 4 foram eliminados pelo facto de faltarem a mais de 8 sessões consecutivamente. Neste sentido, a amostra final passou a ser de 19 sujeitos (12 mulheres e 7 homens) com idade média de $68.7 \pm 4.2$ anos, um peso médio de $66.8 \pm 8.6 \mathrm{Kg}$ e altura média de $1.59 \pm 0.1 \mathrm{~m}$.

As principais características de ambos os grupos (homens e mulheres) estão descritas no Quadro 1.

Quadro 1 - Principais características da amostra [média \pm desvio padrão].

\begin{tabular}{l|c|c|c} 
& Homens & Mulheres & $\mathrm{p}$ \\
\hline Idade (anos) & $68.3 \pm 5.2$ & $69.6 \pm 2.9$ & $\mathrm{~ns}$ \\
\hline Peso $(\mathrm{Kg})$ & $69.1 \pm 12.9$ & $66.7 \pm 7.9$ & $\mathrm{~ns}$ \\
\hline Altura $(\mathrm{m})$ & $1.65 \pm 0.2$ & $1.55 \pm 0.1$ & $\mathrm{~ns}$ \\
\hline $\mathrm{n}$ & $?$ & 12 &
\end{tabular}

Todos os sujeitos da amostra eram voluntários e viviam de forma independente no seu quotidiano. Todos os sujeitos foram informados de todas as possíveis implicações do protocolo experimental, após o que deram o seu consentimento verbal para participarem no estudo.

A presença de patologias crónicas e o uso de medicamentos foram determinados a partir de informação pessoal, assim como, por parte do respectivo médico assistente. Todos os sujeitos eram aparentemente saudáveis e assintomáticos. De igual modo, os medicamentos utilizados pertenciam a grupos farmacológicos considerados como não-influenciadores nos parâmetros avaliados.

Foi pedido a todos os sujeitos amostra para manterem as suas actividades de rotina do dia a dia e para não alterarem o seu nível de actividade física ao longo do programa de treino. Com o objectivo de caracterizar a actividade diária da amostra foi aplicado, nos dois momentos de avaliação, um questionário (37) a todos os idosos da amostra. 


\section{Protocolo de treino}

Todos os sujeitos da amostra foram submetidos a um programa de treino combinado durante 6 meses, que envolveu um programa bi-semanal de "Ginástica de Manutenção" (quartas-feiras e sextas-feiras - 50 min.) e um programa bi-semanal de "Musculação" (terças-feiras e quintas-feiras - 40-50 min.). Todas as sessões foram supervisionadas por um monitor académica e profissionalmente qualificado. As sessões de "Ginástica de Manutenção" foram, genericamente, compostas por: (i) um período de aquecimento de cerca de 10 minutos (caminhar, exercícios calisténicos e exercícios de flexibilidade); (ii) um período de aproximadamente 15 minutos de trabalho muscular (exercícios de força e de flexibilidade); (iii) uma parte aeróbia, num total de cerca de 1/5 da aula (caminhar, dança e jogging); (iv) um período de cerca de 5 minutos com exercícios de coordenação, jogos e equilíbrio; e, por fim, um período de relaxamento/alongamento (cf. estudo 1). $\mathrm{O}$ treino específico de força, incluiu um período de aquecimento estandardizado de baixa intensidade em bicicleta ergométrica (Tectrix, Bike-Max, USA) e/ou remo ergométrico (Concept II, Morrisville, Vermont, USA) e alguns exercícios de alongamento muscular durante cerca de 8-10 minutos.

Posteriormente, existiu um período de exercitação (20-30 minutos) em máquinas comerciais de resistência variável por pesos (Nautilus Sports/ Medical Industries, Independence, USA) e, por fim, efectuou-se um breve período de relaxamento (5-10 minutos) com retorno à calma (caminhar) e alongamento dos principais grupos musculares exercitados. O protocolo de treino de força foi especificamente direccionado para aumentar a força e a massa muscular dos músculos extensores ("leg press" e "leg extension") e flexores do joelho ("seated leg curl"), dos músculos do tronco e membros superiores ("women's double chest", "lateral raise" e "overhead press") e músculos abdominais ("abdominal machine").

Os exercícios da parte superior e inferior do corpo foram efectuados alternadamente a fim de minimizar a fadiga, com um intervalo de repouso de, aproximadamente, 2 minutos. Cada repetição durou 3-6 seg., não existindo um período superior a $2 \mathrm{seg}$. entre as repetições e uma pausa de, pelo menos, 2 minutos entre as 2 séries de 10-12 repetições a 70\% de 1RM. A intensidade do treino foi gradualmente aumentada ao longo das duas primeiras semanas de treino. Assim, o objectivo durante a primeira semana de treino de força foi, para além da determinação da repetição máxima individual (1RM), a familiarização com as máquinas e a consciencialização da correcta realização dos movimentos (técnica de execução e respiração). Nesta fase, o trabalho foi desenvolvido a $60 \%$ de 1 RM. Na segunda semana, a carga foi elevada para $70 \%$ de $1 \mathrm{RM}$, sendo esta a carga imposta até ao final do programa.

Os testes de aferição da carga (1RM) foram realizados de 15 em 15 dias até às primeiras quatro semanas e a cada 4 semanas até ao final do programa. Entre os testes de 1RM, a carga foi aumentada naqueles sujeitos que realizavam confortavelmente 12 ou mais repetições nas 2 séries.

\section{Avaliação da força isocinética}

A força máxima dos músculos extensores e flexores do joelho foi avaliada no membro dominante e não-dominante através de um dinamómetro isocinético (Biodex System 2, USA). Duas velocidades angulares, $60^{\circ} / \mathrm{seg}$. (1.05 rad.seg. ${ }^{-1}$ ) e $180 \%$ seg (3.14 rad.seg. ${ }^{-1}$ ) foram utilizadas, antes (M0) e 6 meses após (M1) o programa de treino, com o sujeito posicionado de acordo com as instruções definidas para este equipamento pela Biodex Medical System, Inc (38).

Após os indivíduos estarem confortavelmente sentados, procedeu-se à colocação das bandas bem ajustadas ao nível do tronco, bacia e coxa, de modo a estabilizar estes segmentos corporais e restringir o mais possível o movimento à flexão e extensão do joelho. O eixo de rotação do dinamómetro foi alinhado com o epicôndilo femoral e a carga de resistência foi colocada cerca de $2 \mathrm{~cm}$ acima do maléolo interno. A referência anatómica angular da articulação do joelho introduzida no dinamómetro foi obtida mediante a utilização de um goniómetro. Os possíveis erros induzidos no torque pela força da gravidade foram corrigidos com base no peso do membro inferior a $0 \%$ seg. e calculados pelo próprio "software" do equipamento. Antes da realização do teste máximo, os sujeitos realizaram um aquecimento estandardizado numa bicicleta ergométrica (Monark, Sweden) durante 5 minutos, a $60 \mathrm{rpm}$, utilizando, para tal, uma carga correspondente a $2 \%$ do peso corporal. Para além disso, antes do teste máximo, os sujeitos tiveram ainda um prévio período de familiarização com o 
dinamómetro através de teste submáximo, que incluiu dez repetições submáximas de extensão/flexão do joelho a $180^{\circ}$ /seg. e cinco repetições a $60 \%$ seg.

Para a avaliação da força máxima, os indivíduos efectuaram cinco repetições máximas a $180^{\circ}$ /seg. e três a $60^{\circ} / \mathrm{seg}$., havendo um período de repouso de 2 minutos entre os testes. Durante o teste, a totalidade do movimento do membro inferior foi requerida desde a posição de flectido $\left(90^{\circ}\right)$ até à máxima extensão possível.

\section{Procedimentos estatísticos}

Procedeu-se a uma análise exploratória dos dados com o objectivo de averiguar a normalidade da distribuição correspondente a cada uma das variáveis em estudo, assim como a presença de "outliers". A análise das diferenças entre os grupos e entre os dois momentos de avaliação foi efectuada a partir de respectivamente, teste de Mann-Whitney e $t$-teste de medidas dependentes. O nível de significância considerado foi de $\mathrm{p}<0.05$.

\section{RESULTADOS}

Os Quadros 2 e 3 representam os valores relativos ao momento máximo, nos dois momentos estudados, de acordo com a variável sexo.

Quadro 2 - Peak Torque (PT, Nm) dos idosos, homens ( $n=7$ ] e mulheres $(n=12$ ), nos momentos inicial e final do treino e respectiva percentagem de alteração na velocidade angular de $180 \%$ seg. (média \pm desvio padrão).

\begin{tabular}{|c|c|c|c|c|c|}
\hline & & ED & END & FD & FND \\
\hline \multirow{2}{*}{$\begin{array}{l}\text { MO } \\
{[\mathrm{Nm}]}\end{array}$} & Homens & $\begin{array}{l}90.8 \\
\pm 25.6\end{array}$ & $\begin{array}{l}77.6 \\
\pm 26.1\end{array}$ & $\begin{array}{l}43.6 \\
\pm 11.5\end{array}$ & $\begin{array}{l}47.6 \\
\pm 14.6\end{array}$ \\
\hline & Mulheres & $\begin{array}{l}59.2^{*} \\
\pm 7.6\end{array}$ & $\begin{array}{l}50.1^{*} \\
\pm 10.8\end{array}$ & $\begin{array}{l}28.0^{*} \\
\pm 7.6\end{array}$ & $\begin{array}{l}28.1^{*} \\
\pm 7.1\end{array}$ \\
\hline \multirow{2}{*}{$\begin{array}{l}\mathrm{M} 1 \\
{[\mathrm{Nm}]}\end{array}$} & Homens & $\begin{array}{l}93.1 \\
\pm 28.9\end{array}$ & $\begin{array}{l}84.1 \\
\pm 29.7\end{array}$ & $\begin{array}{l}48.8 \\
\pm 17.8\end{array}$ & $\begin{array}{l}54.0 \# \\
\pm 13.4\end{array}$ \\
\hline & Mulheres & $\begin{array}{l}61.7^{*} \\
\pm 7.9\end{array}$ & $\begin{array}{l}58.0 * \# \\
\pm ? .5\end{array}$ & $\begin{array}{l}31.2^{*} \\
\pm 6.0\end{array}$ & $\begin{array}{l}35.2^{*} \# \\
\pm 5.7\end{array}$ \\
\hline \multirow{2}{*}{$\begin{array}{l}\% \\
\text { Altera- } \\
\text { ção }\end{array}$} & Homens & $\begin{array}{l}1.9 \\
\pm 11.1\end{array}$ & $\begin{array}{l}7.7 \\
\pm 13.5\end{array}$ & $\begin{array}{l}12.6 \\
\pm 36.7\end{array}$ & $\begin{array}{l}16.6 \\
\pm 15.1\end{array}$ \\
\hline & Mulheres & $\begin{array}{l}4.5 \\
\pm 9.5\end{array}$ & $\begin{array}{l}18.0 \\
\pm 12.9\end{array}$ & $\begin{array}{l}11.7 \\
\pm 27.2\end{array}$ & $\begin{array}{l}29.3 \\
\pm 26.8\end{array}$ \\
\hline
\end{tabular}

$\varepsilon=$ extensão do joelho; $F=$ flexão do joelho; $D=$ membro dominante; $N D=$ membro não-dominante; ${ }^{*}$ homens vs. mulheres; \# MO vs. M1 ( $\left.p<0.05\right)$.
Quadro 3 - Peak Torque (PT, Nm) dos idosos, homens ( $n=7$ ] e mulheres ( $n=12$ ], nos momentos inicial e final do treino e respectiva percentagem de alteração na velocidade angular de 60\% seg. (média \pm desvio padrão)

\begin{tabular}{lccccc}
\hline & & ED & END & FD & FND \\
\hline \multirow{4}{*}{ M0 } & \multirow{2}{*}{ Homens } & 139.1 & 127.9 & 63.8 & 66.1 \\
& & \pm 33.4 & \pm 31.1 & \pm 20.7 & \pm 17.0 \\
\cline { 2 - 6 }$(\mathrm{Nm})$ & \multirow{2}{*}{ Mulheres } & $90.9^{*}$ & $82.8^{*}$ & $46.4^{*}$ & $4.6^{*}$ \\
& & \pm 10.4 & \pm 16.2 & \pm 8.7 & \pm 11.2 \\
\hline \multirow{3}{*}{ M1 } & \multirow{2}{*}{ Homens } & 144.9 & 132.3 & 74.8 & $75.8 \#$ \\
\cline { 3 - 6 }$(\mathrm{Nm})$ & \multirow{2}{*}{ Mulheres } & $95.8^{*}$ & $93.3^{*} \#$ & $47.2^{*}$ & $50.3 \#$ \\
& & \pm 12.3 & \pm 13.8 & \pm 10.3 & \pm 11.4 \\
\hline \multirow{2}{*}{$\%$} & \multirow{2}{*}{ Homens } & 4.5 & 4.3 & 23.3 & 15.5 \\
Altera- & & \pm 9.5 & \pm 14.1 & \pm 37.0 & \pm 13.1 \\
\cline { 2 - 6 } ção & \multirow{2}{*}{ Mulheres } & 5.5 & 13.9 & 9.7 & 23.7 \\
& & \pm 8.1 & \pm 10.5 & \pm 19.6 & \pm 20.7 \\
\hline
\end{tabular}

$\mathcal{E}=$ extensão do joelho; $F=$ flexão do joelho; $D=$ membro dominante; $N D=$ membro não-dominante; ${ }^{*}$ homens vs. mulheres; \# MO vs. M1 ( $\left.p<0.05\right)$.

A análise dos referidos quadros mostra que, no seu conjunto, as mulheres apresentam valores inferiores nas diferentes variáveis relativas ao momento máximo, quer no momento inicial, quer no momento final do treino. No entanto, a percentagem de alteração após o período de treino não foi estatisticamente diferente entre os dois sexos.

Para além disso, é possível constatar que as mulheres, à excepção do membro dominante, quer no movimento de extensão, quer no de flexão em ambas as velocidades avaliadas, apresentam alterações significativas após treino (M1).

Os homens apresentam alterações significativas com o treino na flexão do joelho do membro não-dominante em ambas as velocidades.

\section{DISCUSSÃO}

Os resultados deste estudo sugerem que: $i$ ) um programa combinado de actividade física em, paralelamente às aulas de "Ginástica de Manutenção", seja incluído um trabalho específico de força ("Musculação"), é suficientemente intenso e específico para induzir melhorias nos níveis de força de idosos independentes, aptos e saudáveis de ambos os sexos; ii) apesar das diferenças biológicas entre homens e mulheres, a magnitude das melhorias com o treino é independente do sexo. 
As diferenças observadas no presente estudo, entre homens e mulheres, na força absoluta têm sido também descritas por numerosos autores em diferentes grupos musculares $(14,24,34)$.

No entanto, apesar de vários estudos referirem os níveis mais elevados da força muscular absoluta dos homens, comparativamente às mulheres, nenhum estudo por nós consultado refere uma especificidade de adaptação de acordo com o sexo. Por exemplo, apesar de Tracy et al. (36) encontrarem valores de pré-treino significativamente maiores nos homens, os ganhos musculares relativos do membro exercitado, após treino específico de força durante 9 semanas, foram semelhantes, tanto nos homens, como nas mulheres. De igual modo, Lexell et al. (23) verificaram que, apesar dos níveis iniciais de força entre homens e mulheres serem significativamente diferentes, as respostas ao treino de força foram semelhantes, quer nos extensores do joelho, quer nos flexores do cotovelo. Os resultados deste estudo são concordantes com estes autores, na medida em que os homens apresentaram valores mais elevados em qualquer uma das variáveis estudadas, comparativamente às mulheres, mas a percentagem de alteração nos valores da força muscular com o treino não foi significativamente diferente entre homens e mulheres. Trabalhos anteriores com jovens descrevem que homens e mulheres manifestam aumentos equivalentes na força muscular como resposta ao treino de força. Por exemplo, Castro et al. (7) sugeriram, no seu estudo com jovens, que os valores mais altos na força encontrados nos sujeitos treinados, comparativamente aos não treinados, ocorreram independentemente da variável sexo. De igual modo, Cureton et al. (8) encontraram aumentos semelhantes na força muscular de jovens homens e mulheres após 16 semanas de treino de força.

Neste sentido, a literatura parece ser unânime em considerar que, apesar dos valores absolutos iniciais serem diferentes, as mulheres e os homens idosos adaptam-se de forma similar ao treino de força (16, 22, 36).

Assim, tal como observado no presente estudo, independentemente da idade e do sexo, com estímulos adequados de treino é possível aumentar a força muscular dos músculos exercitados $(15,19,20,22$, 30). Aumentos na força muscular entre 60 a $100 \%$ de 1 RM têm sido observados como resposta ao treino de força (2).

Para além disso, a literatura (para refs. ver 3) tem descrito que os ganhos relativos da força podem estar também relacionados com os valores iniciais, onde os indivíduos com baixos níveis iniciais apresentam uma maior magnitude nos ganhos de força. Por exemplo, Fiatarone et al. (12) encontraram aumentos da força de cerca de $114 \%$ nos membros inferiores após 10 semanas de treino de força em idosos institucionalizados debilitados e com idade bastante avançada. Pelo contrário, no estudo de Berg e Lapp (5), os idosos saudáveis com idade média de 73 anos e que possuíam valores relativamente elevados de força muscular $(111 \mathrm{Nm})$ não apresentaram melhorias após treino.

Os valores iniciais da força da amostra do presente trabalho mostram que os participantes eram relativamente aptos do ponto de vista de força muscular para a média dos indivíduos do mesmo escalão etário (10). De facto, estes valores basais são superiores aos descritos por vários autores $(31,35)$.

Em concordância com a ideia anterior, foram encontrados, nas mulheres, aumentos significativos da força entre o momento final e o inicial no membro não-dominante, quer no movimento de extensão, quer de flexão do joelho em ambas as velocidades angulares estudadas. No entanto, nas mesmas velocidades, no membro dominante não foi observada nenhuma melhoria significativa após treino. Ou seja, as alterações foram mais evidentes naquele membro que, supostamente, é menos utilizado e, como tal, mais fraco.

De igual modo, os idosos homens, que apresentaram valores iniciais de força mais elevados, apenas apresentaram alterações significativas com o treino na flexão do joelho do membro não-dominante em ambas as velocidades, ou seja, no grupo muscular mais débil e no membro supostamente mais fraco. O facto do membro não-dominante apresentar maiores ganhos relativos de força do que o membro dominante é de especial importância, na medida em que faz diminuir o défice bilateral e, como tal, diminuir a probabilidade de lesão (9).

Recentemente, O'Neill et al. (31) encontraram um aumento significativo de cerca de $31 \%$ na força dos músculos extensores do joelho do membro não- 
-dominante, avaliado a 180\% /seg., após treino de força em idosos moderadamente activos com média de idade de $66.3 \pm 1.2$ anos. No presente estudo, nesta variável do momento máximo, foi encontrado, um aumento de, respectivamente, $7.7 \%$ e $18 \%$ após treino nos homens e mulheres. A divergência entre $o$ ganho relativo dos dois estudos pode, entre outros factores, ser justificada pelos valores basais do estudo dos autores que, nesta variável, são ligeiramente inferiores aos observados no presente trabalho. A diferença nos desenhos do protocolo de treino poderá, também, ter contribuído para a divergência das variações entre os estudos. No trabalho de O'Neill et al. (31) foram efectuados treinos tri-semanais de, aproximadamente, 1 hora, sendo realizadas 5 séries de exercícios específicos unilaterais para os extensores e flexores do joelho com 10 repetições cada. No presente estudo, em oposição à maioria dos trabalhos que utilizam treino tri-semanal, os sujeitos treinaram apenas 2 vezes por semana e, dependendo do tipo de exercício e parâmetro avaliado, foram encontrados aumentos da força isocinética entre 2 e $30 \%$. Num estudo recente, Hakkinen et al. (18) encontraram, após treino bi-semanal, aumentos na ordem dos 16 a $28 \%$ na força máxima dos extensores do joelho. Assim, parece que, dependendo do nível inicial, a frequência do treino, pelo menos em idosos sedentários, pode ser tão baixa como 2 vezes por semana, quando a intensidade do treino, tal como no presente estudo, for relativamente elevada e aumentada progressivamente ao longo do período de treino.

Para além disso, é importante salientar que, apesar das alterações no membro dominante após programa de treino combinado não possuírem significado estatístico, esses aumentos foram, em alguns parâmetros, substanciais. Por exemplo, um aumento de $23 \%$ foi observado na força dos homens nos flexores do joelho avaliado a $60^{\circ} /$ seg., o que parece ter um importante significado funcional.

Por outro lado, o facto de não terem existido lesões musculares, nem durante o treino, nem durante as avaliações de 1RM, nem, ainda, na avaliação isocinética da força, reforça a ideia, descrita na literatura (33), de que o treino de força prolongado, de moderada a elevada intensidade, pode ser efectuado com elevada tolerância por sujeitos idosos, com consequente aumento desta capacidade em ambos os sexos.

\section{CONCLUSÃO}

De acordo com os resultados deste estudo, paralelamente a uma actividade física generalizada, o trabalho em máquinas de resistência variável parece ser ideal, uma vez que permite, não apenas a realização controlada do movimento mantendo uma correcta postura, como também ajustar a carga mais apropriada para o grupo muscular e indivíduo em causa, obedecendo, assim, ao princípio da sobrecarga (25). Neste sentido, para além de uma actividade física generalizada, cujo objectivo central se baseia na melhoria de todas as componentes da aptidão física, parece ser necessário um treino específico de força para induzir aumentos significativos da musculatura dos membros inferiores de idosos autónomos e saudáveis, independentemente do sexo. De facto, tratando-se de uma actividade física generalizada, o período de tempo dedicado especificamente ao desenvolvimento da força muscular poderá não ser suficiente para o efeito pretendido (cf. estudo I). Assim, de uma forma geral, o presente estudo mostra que o treino progressivo de força, com intensidade moderada, pode ser efectuado com elevada tolerância por idosos saudáveis, desempenhando um papel importante enquanto estratégia para a manutenção e/ou aumento da sua força dos músculos flexores e extensores do joelho, sendo que a magnitude das melhorias após treino não é significativamente diferente entre os dois sexos.

\author{
CORRESPONDÊNCIA \\ Joana Carvalho \\ Faculdade de Ciências do Desporto \\ e de Educação Física \\ Universidade do Porto \\ Rua Dr. Plácido Costa, 91 \\ 4200.450 Porto \\ Portugal \\ jcarvalho@fcdef.up.pt
}




\section{REFERÊNCIAS BIBLIOGRÁFICAS}

1. Adams K, O'Shea P, O'Shea KL (1999). Aging: its effects on strength, power, flexibility, and bone density. Natl Strength Cond Assoc J 21: 65-77

2. Adams K, Swank AM, Berning JM, Sevene-Adams PG, Barnard KL, Shimp-Bowerman J (2001). Progressive strength training in sedentary older African American women. Med Sci Sports Exerc 33: 1567-1576

3. American College Sports Medicine (1998). Position stand on the recommended quantity and quality of exercise for developing and maintaining cardiorespiratory and muscular fitness, and flexibility in healthy adults. Med Sci Sports Exerc 30: 975-991

4. Aniansson A, Zetterberg C, Hedberg M (1984). Impaired muscle function with aging: a background factor in the incidence of fractures of the proximal end of the femur. Clin Orthop 191: 192-210

5. Berg W, Lapp BA (1998). The effect of a practical resistance training intervention on mobility in independent, community-dwelling older adults. JAPA 6: 18-35

6. Campbell AJ, Robertson MC, Gardner MM, Norton RN, Buchner DM (1999). Falls prevention over 2 years: a randomized controlled trial in women 80 years and older. Age Aging 28: 513-518

7. Castro MJ, McCann DJ, Shaffrath JD, Adams WC (1995). Peak torque per unit cross-sectional area differs between strength-trained and untrained adults. Med Sci Sports Exerc 27: 397-403

8. Cureton KJ, Collins MA, Hill DW, McElhannon J (1988). Muscle hypertrophy in men and women. Med Sci Sports Exerc 20: 1912-1916

9. Davies GJ, Heiderscheit B, Brinks K (2000). Test interpretation. In L.E. Brown (Ed.). Isokinetics in Human Performance. USA: Human Kinetics Publ., 3-24

10. Dvir Z (1995). Isokinetis. Muscle Testing, Interpretation and Clinical Applications. Edinburgh: Churchill Livingstone.

11. Fiatarone MA, Evans WJ (1993). The etiology and reversibility of muscle dysfunction in the aged. J Gerontol 48: 77-83

12. Fiatarone MA, O'Neill EF, Ryan ND, Clements KM, Solares GR, Nelson ME, Roberts SB, Kehayias JJ, Lipsitz LA, Evans WJ (1994). Exercise training and supplementation for physical frailty in very elderly people. $N$ Engl J Med 330: 1769-1775

13. Fleg JL, Lakatta E (1988). Role of muscle loss in the ageassociated reduction in $\mathrm{VO}_{2}$ max. J Appl Physiol 65: $1147-$ 1151

14. Frontera WR, Hughes VA, Lutz KJ, Evans WJ (1991). A cross-sectional study of muscle strength and mass in 45 to 78-yr-old men and women. J Appl Physiol 71: 644-650

15. Hakkinen $\mathrm{K}$, Alen $\mathrm{M}$, Kallinen M, Izquierdo M, Jokelainen K, Lassila H, Malkia E, Kraemer WJ, Newton RU (1998). Muscle CSA, force production, and activation of leg extensors during isometric and dynamic actions in middle-aged and elderly men and women. JAPA 6: 232-247

16. Hakkinen K, Hakkinen A (1995). Neuromuscular adaptations during intensive strength training in middle-aged and elderly males and females. Electrmyogr Clin Neurophysiol 35: $137-147$

17. Hakkinen K, Kraemer WJ, Newton RU, Alen M (2001). Changes in electromyographic activity, muscle fibre and force production characteristics during heavy resistance/power strength training in middle-aged and older men and women. Acta Physiol Scand 171: 51-62
18. Hakkinen K, Pakarinen A, Kraemer WJ, Newton RU, Alen $M$ (2000). Basal concentrations and acute responses of serum hormones and strength development during heavy resistance training in middle-aged and elderly men and women. J Gerontol 55A: B95-B105

19. Higbie EJ, Cureton KJ, Warren GL, Prior BM (1996). Effects of concentric and eccentric training on muscle strength cross-sectional area, and neural activation. J Appl Physiol 81: 2173-2181

20. Kamen G, Sison SV, Duke Du CC, Patten C (1995). Motor unit discharge behavior in older adults during maximaleffort contractions. J Appl Physiol 77: 2648-2658

21. Kaneko M, Morimoto Y, Kimura M, Fuchimoto K, Fuchimoto T (1991). A kinematic analysis of walking and physical fitness testing in elderly women. Can J Sports Sci 16: $223-228$

22. Lemmer JT, Hurlbut DE, Martel GF, Tracy BL, Ivey FM, Metter EJ, Fozard JL, Fleg JL, Hurley BF (2000). Age and gender responses to strength training and detraining. Med Sci Sports Exerc 32: 1505-1512

23. Lexell J, Downham DY, Larsson Y, Bruhn E, Morsing B (1995). Heavy-resistance training for Scandinavian men and women over seventy: short-and long-term effects on arm and leg muscles. Scand J Med Sci Sports 5: 329-341

24. Lindle RS, Metter EJ, Lynch NA, Fleg JL, Fozard JL, Tobin J, Roy TA, Hurley BF (1997). Age and gender comparisons of muscle strength in 654 women and men aged 20-93 yr. J Appl Physiol 83: 1581-1587

25. Lord SR, Ward JA, Williams P, Strudwick M (1995). The effects of a 12-month exercise trial on balance, strength, and falls in older women: a randomized controlled trial. $J$ Am Geriatr Soc 43: 1198-1206

26. Mazzeo RS, Tanaka H (2001). Exercise prescription for the elderly. Current recommendations. Sports Med 31: 809-818

27. McFadyen BJ, Winter DA (1988). An integrated biomechanical analysis of normal stair ascent and descent. J Biomech 21: 733-744

28. Miller JP, Pratley RE, Goldberg AP, Gordon P, Rubin M, Treuth MS, Ryan AS, Hurley BF (1994). Strength training increases insulin action in healthy 50 -to 65 -yr-old men. $J$ Appl Physiol 77: 1122-1127

29. Millington PJ, Myklebust BM, Shambes GM (1992). Biomechanical analysis of the sit-to-stand motion in elderly persons. Arch Phys Med Rehab 73: 609-617

30. Narici M, Hoppeler H, Kayser B, Landoni L, Claasen H, Gavardi C, Conti M, Cerretelli P (1996). Human quadriceps cross-sectional area, torque, and neural activation during 6 months strength training. Acta Physiol Scand 157: 175-186

31. O'Neill DET, Thayer RE, Taylor AW, Dzialoszynski TM, Noble EG (2000). Effects of short-term resistance training on muscle strength and morphology in the elderly. JAPA 8: 312-324

32. Puggaard L, Pedersen HP, Sandager E, Klitgaard H (1994). Physical conditioning in elderly people. Scand J Med Sci Sports 4: 47-56

33. Pyka G, Linderberger E, Charette S, Marcus R (1994) Muscle strength and fiber adaptations to a year-long resistance training program in elderly men and women. $J$ Gerontol 49: M22-M27 
34. Rice CL, Cunnigham DA, Paterson DH, Lefcoe MS.

(1989). Strength in an elderly population. Arch Physiol Med Rehab 70: 391-397

35. Salem GJ, Wang M-Y, Young JT, Marion M, Greendale GA (2000). Knee strength and lower- and higher-intensity functional performance in older adults. Med Sci Sports Exerc 32: 1679-1684

36. Tracy BL, Ivey FM, Hurlbut D, Martel GF, Lemmer JT, Siegel EL, Metter EJ, Fozard JL, Fleg JL, Hurley BF (1999). Muscle quality II. Effects of strength training in 65- to 75yr-old men and women. J Appl Physiol 86: 195-201

37. Voorrips LE, Ravelli ACJ, Dongelmans PCA, Deurenberg P, van Staveren WA (1991). A physical activity questionnaire for the elderly. Med Sci Sports Exerc 23: 974-979

38. Wilk K (1991). Isokinetic Testing - Setup and Positioning. In Biodex System II Manual, Applications/Operations. Biodex Medical System, Inc, New York, USA. 